\title{
Bioinformatics of Rhizoma Curcumae against Gastric Carcinoma Grafted on Mice
}

\author{
Huanping Lin ${ }^{\mathrm{a}}$, Xiaoping Wang ${ }^{b^{*}}$, Qiaoxia Wang ${ }^{\mathrm{c}}$, Bing $\mathrm{Xu}^{\mathrm{d}}$ and $\mathrm{Na}$ Chang $^{\mathrm{e}}$ \\ Key lab of Molecular Pathology \\ Shaanxi University of Chinese Medicine \\ Xianyang 712046, China

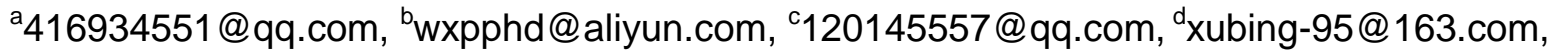 \\ 825865603@qq.com
}

*The Corresponding author

Keywords: Rhizoma curcumae; Gastric cancer; Apoptosis; Signal transduction; Cytotoxicity.

\begin{abstract}
Objective: The study is intended to explore the anti-tumor effect of the rhizoma curcumae in treatment of gastric cancer. Methods: Kunming mice grafted with a mouse gastric adenocarcinoma cell line MFC was used as the experimental model. The mice were divided into four groups, one control and the other three different drug administration experimental groups. The anti-tumor effect was assessed by tumor size, apoptotic indices (AI) and the expression of VEGF, STAT3 and HIF-1 $\alpha$ in tumor tissues. Results: Compared with controls, tumor size and weigh were significantly inhibited with the rhizoma curcumae decoction treatment $(\mathrm{P}<0.05)$. AI in gastric cancer grafted mice was significantly increased in the rhizoma curcumae decoction group. The expression of VEGF, STAT3 and HIF-1 $\alpha$ in tumor tissues were down-regulated after treated with rhizoma curcumae decoction. Conclusion: The anti-tumor effect of gastric cancer cell growth in vivo by rhizoma curcumae is related with the induction of the cell apoptosis and down-regulation of VEGF, STAT3 and HIF-1 $\alpha$ signal transduction system.
\end{abstract}

\section{Introduction}

In clinic studies, Chinese herb rhizoma curcumae had been found to have effect on pre-malignant lesion, especially on gastric diseases $[1,2]$. The rhizoma curcumae decoction might inhibit gastric carcinoma cell proliferation and cause tumor cell death. Apoptosis plays a crucial role in the proliferation and turnover of cells in various tumors. It has been clear that its extent is often enhanced in tumor by many anticancer drugs, such as cytotoxic drugs, hormone, or some Chinese herbal medicine [3-5]. Researches indicated that Chinese herbs could enhance apoptosis of human gastric cancer grafted in mice [5,6].

Hypoxia-inducible factor-1a (HIF-1a) mediates hypoxic and non-hypoxic signaling pathways, and plays a crucial role in cancer progression. Over-expression of HIF- $1 \alpha$ has been found in gastroenterological cancers and it is implicated in treatment resistance and poor prognosis in the hypoxic region around cancer [7]. Vascular endothelial growth factor (VEGF) has been proved to be a major inducer of angiogenesis, lymph angiogenesis and vasculogenesis in cancer [8]. The JAK/STAT3 signaling cascade play essential roles in promoting cancer cell survival, proliferation, angiogenesis and tumor metastasis [9].

Based on the previous studies, we presumed whether the rhizoma curcumae could affect the apoptotic indices of gastric cancer grafted onto mice and the expression of VEGF-STAT3-HIF-1 $\alpha$ signal transduction in gastric cancer, further confirming the anti-tumor mechanism of the Chinese herb rhizoma curcumae. 


\section{Materials and Methods}

Mice and Antibody Reagents. Forty 6-8 weeks old female Kunming mice (weight 18-22 g) and a mice gastric carcinoma cell line MFC were obtained from the Fourth Military Medical University. The mice were subcutaneously grafted with the MFC cells. The tumor transplantation procedure was described previously. Rabbit anti-mouse HIF-1 $\alpha$, STAT3 and VEGF polyclonal antibody were purchased from Santa Cruz Biotechnology, Inc (Santa Cruz, CA, USA). EnVisionTM kits were purchased from Dako Corp (Carpinteria, CA, USA).

Drugs. The rhizoma curcumae decoction consists of Codonopsis pilosula (Franch) Nannf., Atractylodes macrocephala koidz, Poria cocos (Schw.) Wolf, Glycyrrhiza uralensis Fisch. The concentration of the rhizoma curcumae decoction was $240 \mathrm{~g} / \mathrm{L}$.

Administration. After grafting the mice were randomly divided into 4 groups, one control and the other three experimental groups assigned to receive the rhizoma curcumae decoction. Each animal in the three experimental groups was given $2.0 \mathrm{~mL}, 1.0 \mathrm{~mL}$ and $0.5 \mathrm{~mL}$ of the rhizoma curcumae decoction by gastric perfusion every day over a 60-day period beginning at 1 st day after grafting. The control animals received normal saline according to the same schedule. Animals were killed 61 days after being grafted.

Assessment of Tumor Growth. The effect of therapy was assessed by two ways: (1) tumor size was measured twice a week by multiplying two perpendicular diameters. (2) tumor weight was determined immediately by electron balance after the animals were killed. The tumor tissues were fixed in $10 \%$ formalin, embedded in paraffin and cut into $5 \mu \mathrm{m}$ sections coated on the slides for staining.

Detection of Apoptosis. For detection of apoptotic cells, apoptotic indices were examined by the terminal deoxynucleotidyl transferase-mediated deoxyuridine triphosphate fluorescence nick end labeling (TUNEL) method and flow cytometry analysis. (1) TUNEL: In situ cell death detection Kit POD (Roche Applied Science, Indianapolis, USA) was used to detect the apoptotic cell. The procedures were referred to the kit protocol. The positive cells were identified, counted and analyzed under the light microscope. Non-necrotic zone was selected in the tissue section and images were sent to computer by AEC camera (ZEISS, Jena, Germany). 10 image at least 1000 cells were selected on the screen, positive ratio analyzed by KS400 Video Image Digital Analysis System (ZEISS, Jena, Germany). (2) Flow cytometry analysis: Propidium iodide (PI) staining was used for flow cytometry detection of apoptosis. $10^{6}$ cells from each of the sample were treated with RNase and stained with PI. The apoptotic cells having DNA strand breaks that had been labeled were measured on a flow cytometer (FACSCalibur, Becton Dickinson, USA). The data from $10^{6}$ cells/sample were collected, stored, and analyzed using CELLQUEST (Becton Dickinson USA) and ModFIT LT for mac V1.01 software (Becton Dickinson, USA).

Immunostaining Methods. All sections were deparaffinized and rehydrated with graded alcohols. Endogenous peroxidase was then blocked with $3 \mathrm{~mL} / \mathrm{L} \mathrm{H} 2 \mathrm{O} 2$ diluted in methanol for 30 $\mathrm{min}$ at room temperature. Antigen retrieval was performed by treating the slides in citrate buffer in a microwave for $10 \mathrm{~min}$. The slides were incubated in a moist chamber with HIF-1 $\alpha$, STAT3 or VEGF rabbit polyclonal antibody $(1: 100)$ at $4{ }^{\circ} \mathrm{C}$ overnight. After a complete wash in phosphate buffered saline (PBS), the slides were incubated with horseradish peroxidase labeled goat antimouse antibody $(1: 100)$ for $45 \mathrm{~min}$ at $37^{\circ} \mathrm{C}$. After a complete wash in PBS, the slides were developed in $0.5 \mathrm{~g} / \mathrm{L}$ freshly prepared 3,3'-diaminobenzedine solution (DAB, Sigma Co, St.Louis, Mo, USA) for $8 \mathrm{~min}$, and then counterstained with hematoxylin, dehydrated, air dried, and mounted. Normal mouse IgG was used to substitute for the primary antibody as a negative control. Only distinctive intranuclear or intra-cytoplasm immunoreactivity was considered positive. In each case, more than 1000 cells were counted and the percentage of immunoreactivity was independently determined. Image $\mathbf{J}$ analysis software was used to acquire the optical density data from the staining sections.

Statistical Analysis. All data were showed as means \pm S.D. The apoptosis indexes were assessed by $\chi^{2}$ test. The Student's $t$ test was conducted to explain the significant differences between tumor volumes of each animal group. Statistically significance was considered as $\mathrm{P}<0.05$. 


\section{Results}

Inhibition of Tumor Growth by Rhizoma Curcumae Decoction. Compared with the control group, tumor growth (size and weight) was significantly inhibited by treatment with the rhizoma curcumae decoction $(\mathrm{P}<0.05$, Table 1$)$. The results showed that the higher the concentration of rhizoma curcumae decoction, the less the tumor weight and size. There was a significant difference between the rhizoma curcumae and control group.

Table 1 Rhizoma curcumae induced effects on gastric cancer

\begin{tabular}{|c|c|c|}
\hline Groups Treatment & Tumor weight(g) & Tumor size $\left(\mathrm{mm}^{3}\right)$ \\
\hline $\begin{array}{l}\text { High-dose rhizoma } \\
\text { curcumae decoction }\end{array}$ & $0.54 \pm 0.35^{\mathrm{a}}$ & $262.54 \pm 43.26^{\mathrm{a}}$ \\
\hline $\begin{array}{l}\text { Middle-dose rhizoma } \\
\text { curcumae decoction }\end{array}$ & $0.75 \pm 0.22^{\mathrm{b}}$ & $327.46 \pm 35.46^{\mathrm{b}}$ \\
\hline $\begin{array}{l}\text { Low-dose rhizoma } \\
\text { curcumae decoction }\end{array}$ & $0.99 \pm 0.34^{\mathrm{c}}$ & $577.38 \pm 62.42^{\mathrm{c}}$ \\
\hline Control & $1.13 \pm 0.25$ & $580.27 \pm 71.58$ \\
\hline
\end{tabular}

Induction of Tumor Cell Apoptosis by Rhizoma Curcumae Decoction. Apoptotic index (AI) in mice loaded with gastric cancer cells was significantly elevated to $18.33 \pm 2.47 \%$ by TUNEL method and $17.88 \pm 5.73 \%$ FACScan in the rhizoma curcumae decoction treatment group, compared with the controls (TUNEL: $2.89 \pm 1.75 \%, \mathrm{P}<0.05$; FACScan: $4.34 \pm 2.03 \%, \mathrm{P}<0.05$ ). But there was no significant difference between low-dose rhizoma curcumae decoction treated group and control group by using either TUNEL method or flow cytometry analysis (Table 2).

Table 2 Rhizoma curcumae induced apoptosis on gastric cancer cells

\begin{tabular}{|c|c|c|}
\hline Groups Treatment & TUNEL Apoptotic index & $\begin{array}{c}\text { FACScan Apoptotic } \\
\text { (AI)(\%) }\end{array}$ \\
\hline $\begin{array}{c}\text { High-dose rhizoma } \\
\text { curcumae decoction }\end{array}$ & $18.33 \pm 2.47^{\mathrm{a}}$ & $17.88 \pm 5.73^{\mathrm{a}}$ \\
\hline $\begin{array}{c}\text { Middle-dose rhizoma } \\
\text { curcumae decoction }\end{array}$ & $10.05 \pm 5.42^{\mathrm{b}}$ & $9.85 \pm 4.65^{\mathrm{b}}$ \\
\hline $\begin{array}{c}\text { Low-dose rhizoma } \\
\text { curcumae decoction }\end{array}$ & $3.12 \pm 1.54^{\mathrm{c}}$ & $5.68 \pm 2.26^{\mathrm{c}}$ \\
\hline Control & $2.89 \pm 1.75$ & $4.34 \pm 2.03$ \\
\hline \multicolumn{2}{|c|}{${ }^{\mathrm{a}} \mathrm{P}<0.05,{ }^{\mathrm{b}} \mathrm{P}<0.05,{ }^{\mathrm{c}} \mathrm{P}>0.05$, vs control group d. } \\
\hline
\end{tabular}

Expression of VEGF, STAT3 and HIF-1 $\alpha$ in Gastric Cancers. HIF-1 $\alpha$, STAT3 and VEGF immunoreactivities were detected in 40 mice gastric tumors. HIF-1 $\alpha$ proteins were mainly presenting a nuclei staining, while STAT3 and VEGF mainly stained in the cell cytoplasm. The optical density (OD) value of HIF-1 $\alpha$, STAT3 and VEGF in rhizoma curcumae herbs treated groups were significantly lower than that in control $(\mathrm{P}<0.05$, Table 3$)$.

Table 3 Expression of VEGF, STAT3 and HIF-1 $\alpha$ in gastric cancers

\begin{tabular}{|c|c|c|c|}
\hline Groups Treatment & $\begin{array}{c}\text { VEGF Optical } \\
\text { density (OD) }\end{array}$ & $\begin{array}{c}\text { STAT3 Optical } \\
\text { density (OD) }\end{array}$ & $\begin{array}{c}\text { HIF-1a Optical } \\
\text { density (OD) }\end{array}$ \\
\hline High-dose rhizoma & $0.018 \pm 0.065^{\mathrm{a}}$ & $0.024 \pm 0.012^{\mathrm{a}}$ & $0.012 \pm 0.008^{\mathrm{a}}$ \\
\hline
\end{tabular}




\begin{tabular}{|c|c|c|c|}
\hline curcumae decoction & & & \\
\hline $\begin{array}{c}\text { Middle-dose rhizoma } \\
\text { curcumae decoction }\end{array}$ & $0.025 \pm 0.032^{\mathrm{b}}$ & $0.039 \pm 0.005^{\mathrm{b}}$ & $0.023 \pm 0.014^{\mathrm{b}}$ \\
\hline $\begin{array}{c}\text { Low-dose rhizoma } \\
\text { curcumae decoction }\end{array}$ & $0.051 \pm 0.007^{\mathrm{c}}$ & $0.052 \pm 0.014^{\mathrm{c}}$ & $0.038 \pm 0.006^{\mathrm{c}}$ \\
\hline Control & $0.050 \pm 0.015$ & $0.051 \pm 0.004$ & $0.036 \pm 0.022$ \\
\hline \multicolumn{2}{|r|}{${ }^{\mathrm{a}} \mathrm{P}<0.05,{ }^{\mathrm{b}} \mathrm{P}<0.05,{ }^{\mathrm{c}} \mathrm{P}>0.05$, vs control group d. }
\end{tabular}

\section{Discussion}

Most concerns have focused on cancer immunity which aims to elicit hosts' immune protection against tumor cells. $80 \%$ of primary liver cancers express a high rate of alpha-fetoprotein (AFP), which may act as a target for cancer therapy [3-5]. AFP is an oncofetal antigen during HCC progression which could induce weak reproducible antitumor effect. Therefore, a vaccine specifically targeting AFP is desirable. AFP oncofetal antigen is essential to the promotion and maintenance of cancer cell transformation, which could be an ideal target antigen for AFP vaccine [6,7]. However, AFP immunization has been restricted by improper antigen-specific immune reaction. Therefore, therapies to generate more potential immunotherapy need to be completely explored.

Gastric carcinoma is one of the most common malignant gastrointestinal carcinoma in the world. At present gastric carcinoma is still detected later in most patients throughout the world, and even with curative resection, they remain at a high risk of relapse and mortality. Thus, there is a great need for effective adjuvant therapy for patients with gastric carcinoma. Our previous clinic studies suggested that Chinese herbal recipe rhizoma curcumae have therapeutic effects on gastric premalignant lesion, with increasing the reversal of the atrophic gastritis, decreasing the recurrence and improving the life quality [1,2]. Because of its lower toxic side-effect compared with chemical therapy, it is worth to make a further research on its anti-cancer mechanism.

Similar to the other malignant tumors, gastric carcinoma is always accompanying with abnormal cell proliferation and differentiation, as well as with abnormal apoptosis [5,6]. The enhanced induction of apoptosis in human gastric carcinoma cells can be observed after treatment with 5Fluorouracil, Cisplatin, arsenous oxide and so on $[5,6,10]$. These data suggest that inducing cancer cell apoptosis should be a therapeutic method for gastric carcinoma. The present study indicated that tumor growth was significantly inhibited by treatment with the rhizoma curcumae decoction. TUNEL method and cytometry analysis clarified that rhizoma curcumae decoction could enhance apoptosis. The results suggest that the mechanism of the inhibition of gastric cancer cells in vivo by rhizoma curcumae herbs is related with inducing apoptosis.

The VEGF-STAT3-HIF-1 $\alpha$ signal transduction pathway plays an important role in the development and progression of gastrointestinal cancers [11]. Our results suggested that rhizoma curcumae herbs could down-regulate the expression of VEGF-STAT3-HIF-1 $\alpha$ signal transduction proteins in gastric cancer, thus inhibiting the growth of tumor cells.

\section{Summary}

In conclusion, rhizoma curcumae decoction inhibited gastric cancer cell growth. The anti-tumor effect of rhizoma curcumae herbs lies in inducing gastric cancer cells apoptosis and downregulating the expression of VEGF-STAT3-HIF-1 $\alpha$ signal transduction protein in tumor cells. The detailed molecular mechanism of rhizoma curcumae herbs inhibiting gastric cancer cells still needs further investigation.

\section{Acknowledgments}

This work is supported by Scientific Research Program Funded by Shaanxi University of Chinese Medicine and Shaanxi Provincial Education Department (No.2007JK233, 2009xj02, 2010JK484, 
14JS025), the Scientific Research Program of Shaanxi Administration of Traditional Chinese Medicine (No.15-SCJH001) and the Natural Science Basic Research Plan in Shaanxi Province of China (No.2016JM8023, 2016JM8150).

\section{References}

[1] H.P. Lin, S.C. Li, Protective effects of rhizoma curcumae on acute gastric mucous jnjury on rat, Shaanxi Journal of Traditional Chinese Medicine. 28 (2007) 1422-1423.

[2] S.C. Li, F. Wang, H.P. Lin, X.Y. Zhou, Effects of rhizoma curcumae on the secretion of serum gastrin and motilin in chronic atrophic gastritis, Jiangxi Journal of Traditional Chinese Medicine. 38 (2007) 68-69.

[3] T. Matsunaga, Y. Tsuji, K. Kaai, S. Kohno, R. Hirayama, D.H. Alpers, et al. Toxicity against gastric cancer cells by combined treatment with 5-fluorouracil and mitomycin c: implication in oxidative stress, Cancer Chemother. Pharmacol. 66 (2010) 517-526.

[4] L. Qiao, B.C. Wong, Targeting apoptosis as an approach for gastrointestinal cancer therapy, Drug Resist. Updat. 12 (2009) 55-64.

[5] A.G. Zhao, H.L. Zhao, X.J. Jin, J.K. Yang, L. Tang, Effects of Chinese Jianpi herbs on cell apoptosis and related gene expression in human gastric cancer grafted onto nude mice, World J. Gastroenterol. 8 (2002) 792-796.

[6] J. Li, G.Z. Sun, H.S. Lin, Y.X. Pei, X. Qi, C. An, et al. The herb medicine formula "Yang Wei Kang Liu" improves the survival of late stage gastric cancer patients and induces the apoptosis of human gastric cancer cell line through Fas/Fas ligand and Bax/Bcl-2 pathways, Int. Immunopharmacol. 8 (2008) 1196-1206.

[7] G.L. Semenza, Hypoxia-inducible factors: mediators of cancer progression and targets for cancer therapy, Trends Pharmacol. Sci. 33 (2012) 207-214.

[8] N. Pore, Z. Jiang, A. Gupta, G. Cerniglia, G.D. Kao, A. Maity, EGFR tyrosine kinase inhibitors decrease VEGF expression by both hypoxia-inducible factor (HIF)-1-independent and HIF-1dependent mechanisms, Cancer Res. 66 (2006) 3197-3204.

[9] N. Li, I. Grivennikov Sergei, M. Karin, The unholy trinity: inflammation, cytokines, and STAT3 shape the cancer microenvironment, Cancer Cell. 19 (2011) 429-431.

[10]D. Korbakis, A. Scorilas, Treatment of gastric cancer cells with 5-fluorouracil/leucovorin and irinotecan induces distinct alterations in the mRNA expression of the apoptosis-related genes, including the novel gene BCL2L12, Tumour Biol. 30 (2009) 100-107.

[11] K.M. Dodd, J. Yang, M.H. Shen, J.R. Sampson, A.R. Tee. mTORC1 drives HIF-1 $\alpha$ and VEGFA signaling via multiple mechanisms involving 4E-BP1, S6K1 and STAT3, Oncogene. 34 (2015) 2239-2250. 